\title{
Analysis on Green Airport Pollution Rating Model based on Analytic Hierarchy Process and Neural Network
}

\author{
Xin Liu \\ Qingdao University of Technology, Qingdao, Shandong 266300, China
}

Keywords: Green Airport Pollution, Analytic Hierarchy Process, Neural Network, Analysis.

\begin{abstract}
In this paper, we conduct research on the green airport pollution rating model based on analytic hierarchy process and neural network. Environmental protection departments should summarize main airport, regional airport as soon as possible the noise characteristics and scope of reference foreign noise control experience, as soon as possible to establish sound insulation measures such as noise removal and control standards, and provide guidance for relocation and sound insulation measures. This paper combines the state-of-the-art methodologies on the corresponding research result to propose the new methodology on the modelling procedures that is innovative. We review the mathematical model for the corresponding issues to serves as the basis that is meaningful.
\end{abstract}

\section{Introduction}

Aviation industry is rapidly growing global industry as economic globalization in recent years been facilitated, which makes the scope of international cooperation is becoming more and more widely. Governments recognize the importance of the airport as the airline industry is one of the key industry to promote the development of the economic stability and the airport as the world's people, logistics, information, capital, knowledge, and the hub of the cultural exchange, both at home and abroad have made a significant impact on regional economy. To build a scientific use of evaluation index system of green airport, practical significances are reflected from the listed aspects [1-2].

The airport managers, the establishment of evaluation index system of green airport, from the soft indexes of management, not only to focus on building hard indexes such as that provided the foundation for comparison for each management direction.

For passengers to consumers, the establishment of evaluation index system of green at the airport that can make the passengers to the airport's green degree has a more specific understanding, and through the improvement of the system to understand.

For building designers, the evaluation index system of green airport for each indicator has a detailed comparison and quantitative help designers from the angle of ecology, environmental protection, to optimize the design of the airport, in the design of the airport building phase implementation airport ecological, environmental protection, comprehensive combination of the factors such as management, service, and make green inspection of the airport building.

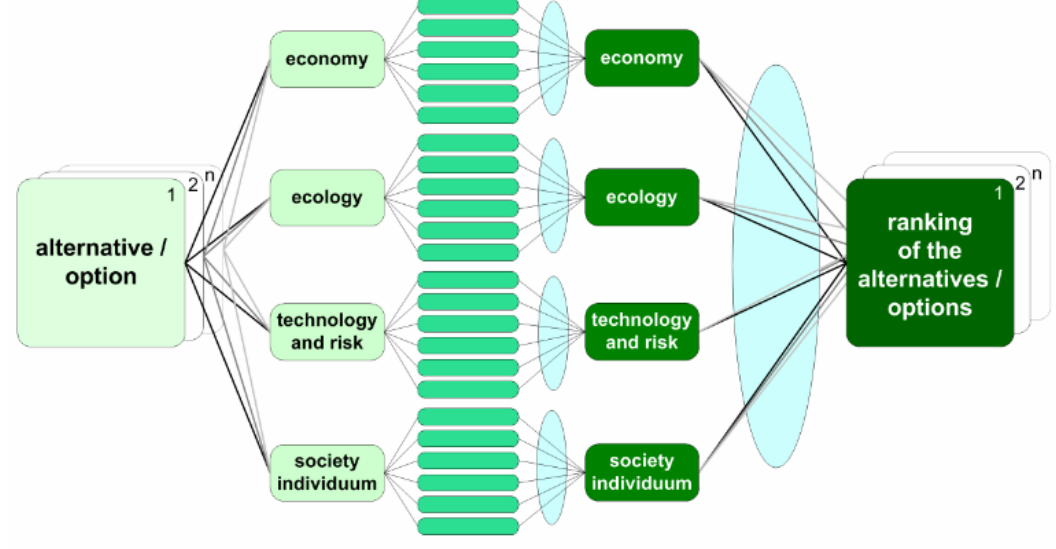

Fig. 1The Green Airport Pollution Rating Model Reference Pattern 
Under this basis, in this paper, we conduct research on the green airport pollution rating model based on analytic hierarchy process and neural network. In the later parts, we will discuss in detail.

\section{The Proposed Methodology}

\subsection{Analytic Hierarchy Process.}

Analytic hierarchy process (AHP) is a combination of qualitative and quantitative analysis method of multi-objective decision analysis method. The main ideas of the law by the complex problem is decomposed into several levels and a number of factors, the paper compares two important degree between the two indicators judgment, and to establish judgment matrix, by computing the judgment matrix largest eigenvalues and corresponding eigenvectors can give different weights of importance degree that provide the basis for the selection of the best solution [3].

Are derived from the analytic hierarchy process (AHP) after years of development, improve the analytic hierarchy process (AHP) and fuzzy analytic hierarchy process (AHP) and extension methods such as analytic hierarchy process (AHP) and grey AHP, and according to the actual situation of each has its applicable scope. Improved analytic hierarchy process (AHP), fuzzy analytic hierarchy process (AHP) and extension of fuzzy analytic hierarchy process (AHP) is based on judgment matrix under the condition of not sure, judging by improving scale is easier to help decision makers to construct good quality judgment matrix and grey hierarchical analysis law is the combination of grey system theory with analytic hierarchy process, the grey theory through establishing model, structure matrix, weight calculation and result evaluation of the whole process. In the figure 2, we show the sample.

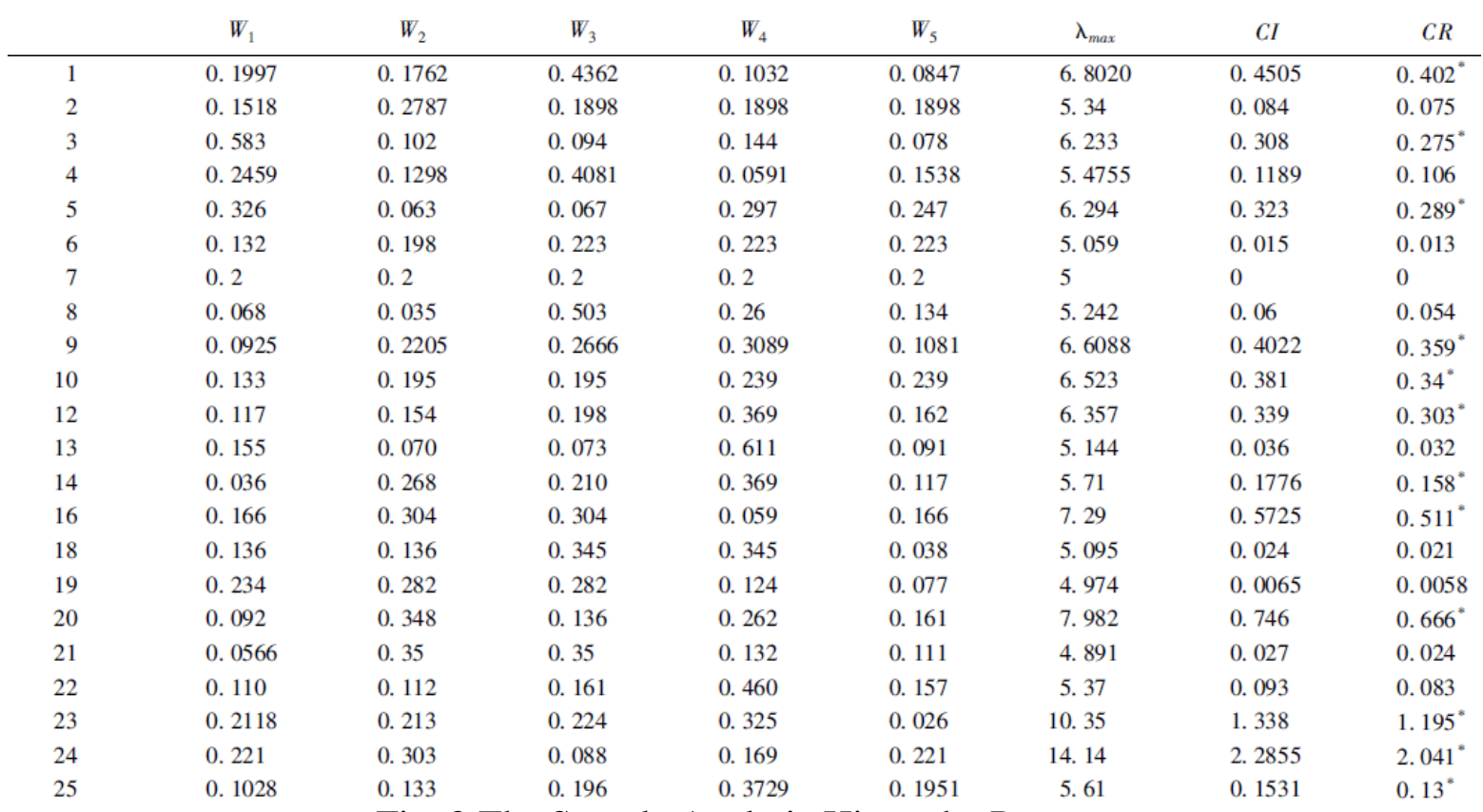

Fig. 2 The Sample Analytic Hierarchy Process

\subsection{The Neural Network Evaluation Model.}

Neural network is used to forecast model of the basic requirements are: the good convergence, real-time and certain generalization ability, etc. Before the training of neural network, must first determine the type selection of neural network. The neural network types many, must according to the nature of the problem and task requires to selects the appropriate network type. Inappropriate increase may lead to neural network training can't even convergence. In predictive modeling, application is more a RBF network, it to a certain extent, to overcome the local optimal BP network existence, the problem of slow training speed and good approximation of nonlinear performance of the model.

Modeling method of neural network prediction model can be summarized as the follows. (1) Based on a modular model. The use of multiple models to reflect the change of the dynamic characteristics, 
reduces the modeling range of local model, improve the accuracy of modeling that has the very good interpolation and extrapolation properties. (2) Based on the prediction deviation compensation model. Neural network prediction model using multi-step prediction method to predict the future output value, in order to overcome the uncertainty of the system, enhance the system robustness. But in the process of modeling the model error, this is bound to affect the accuracy of prediction. (3) Learning algorithm of neural network model. Based on the construction of learning algorithm of single hidden layer neural network structure to approximate the dynamic behavior of a class of general complex multivariable process, the construction of learning algorithm uses the mapping tracking technology, has reliable accuracy. (4) Based on linear model and the combination of neural network model. Owing with nonlinear neural network, it is used to solve the optimization controller, not only difficult to obtain accurate analytical solution, and in a short time enough to allow algorithm converges to the optimal solution is also more difficult that in order to solve the shortage, many literature numerical optimization method to improve the optimization speed and performance of the controller and the procedure can be generally summarized as the follows [4].

$$
\begin{gathered}
J\left(u(k), \cdots, u\left(k+N_{u}-1\right)\right)=\sum_{j=N_{1}}^{N_{2}}\left[y_{r}(k+j)-\right. \\
\left.y_{p}(k+j)\right]^{2}+\rho \sum_{j=1}^{N_{u}}[\Delta u(k+j)]^{2} \\
\text { s.t. }\left\{\begin{array}{l}
\Delta u(k+j)=u(k+j)-u(k+j-1), \quad j=0,1, \ldots, m-1 \\
u^{\min } \leq u(k+j) \leq u^{\max } \\
y^{\min } \leq y_{p}(k+j) \leq y^{\max }
\end{array}\right.
\end{gathered}
$$

An algorithm is especially the pros and cons of online algorithm are usually composed of two standards: amount of basic calculation and convergence stability. This article has through the neural network inverse, and as much as possible to reduce the search interval width, thus reduce the online computation which could be generally shown as the figure 3 .

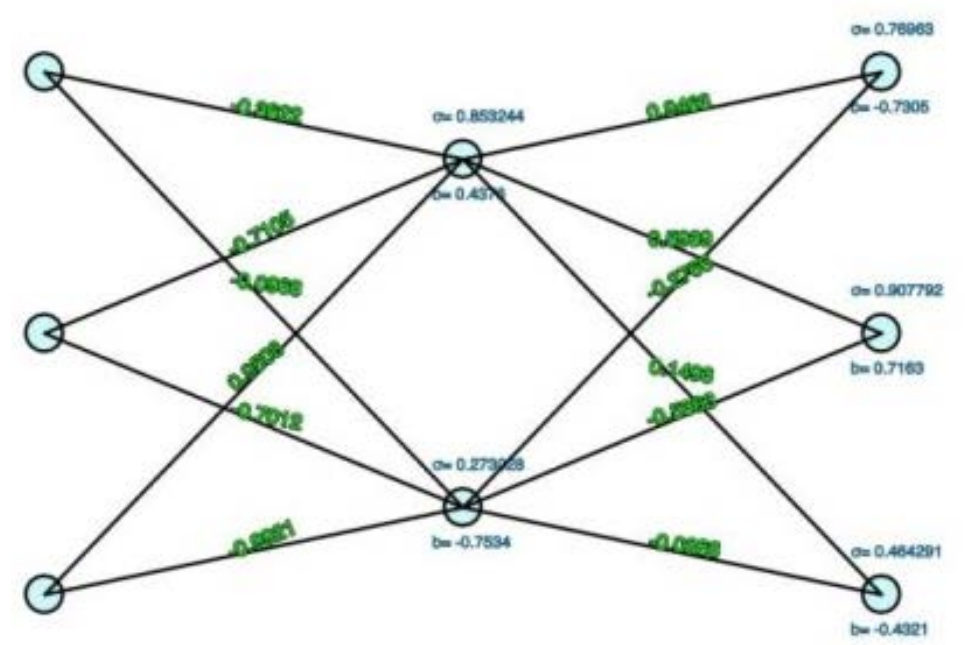

Fig. 3The Neural Network Evaluation Model Demonstration

\subsection{Airport Pollution Rating Model.}

Airport air pollution there are two main reasons: one is the airport during the initial period of the construction, construction conditions and construction vehicle, and lime, fly ash and other material transport and general storage caused by dust of total suspended particulate matter in the surrounding environment more than the ambient air quality standards. Second it is to the airport after put into the operation, the plane's engine exhaust gases from the combustion chamber, the airport boiler flue gas emissions and vehicle exhaust emissions from the airport and so on as these gases contain more carbon compounds, nitrogen compounds and suspended particles. 
Environmental protection departments should summarize main airport, regional airport as soon as possible the noise characteristics and scope of reference foreign noise control experience, as soon as possible to establish sound insulation measures such as noise removal and control standards, provide guidance for relocation and sound insulation measures. For this goal, we summarize the existing countermeasures as the follows to provide the solution [5].

ADMS-Airport model is to calculate air quality evaluation model of pollutant concentration around the airport. ADMS is to use the input changes per hour of the meteorological data, emission and pollution data to calculate the concentration of pollution. The model considers both the topography influence on wind speed, and the building of influence of air diffusion.

LASPORT model is dedicated to research and development of the airport in Germany, the model based on Lagrange model for diffusion model, an aircraft, ground support equipment, such as the airport parking lot vehicle related emission standards and calculation tool.

EDMS diffusion model is the core of gauss diffusion equation. Commonly used point source summation formula of that line source dispersion model application EDMS5.1 model to the capital airport aircraft and ground equipment discharge listing and to establish a method to calculate discharge atmospheric pollutants.

\section{Summary}

In this paper, we conduct research on the green airport pollution rating model based on analytic hierarchy process and neural network. For airport, energy-saving technological transformation and building energy management system, the two complement each other, energy saving is a link in the whole energy management system and a means of execution, to achieve perfection, still need to build energy management system, implement the systematic and fine management of energy. This paper combines the state-of-the-art methodologies on the corresponding research result to propose the new methodology on the modelling procedures that is innovative.

\section{References}

[1]. Di Menno, di Bucchianico A., et al. "[Air pollution in an urban area nearby the Rome-Ciampino city airport]." Epidemiologia e prevenzione 38.3-4 (2013): 244-253.

[2]. Isakov, V., et al. "Development of Model-Based Air Pollution Exposure Metrics for Use in Epidemiologic Studies." Air Pollution Modeling and its Application XXIII. Springer International Publishing, 2014. 19-24.

[3]. Sørensen, Mette, et al. "Exposure to long-term air pollution and road traffic noise in relation to cholesterol: A cross-sectional study." Environment international 85 (2015): 238-243.

[4]. Kimbrough, Sue, et al. "Long-term continuous measurement of near-road air pollution in Las Vegas: seasonal variability in traffic emissions impact on local air quality." Air Quality, Atmosphere \& Health 6.1 (2013): 295-305.

[5]. Stansfeld, Stephen A. "Noise effects on health in the context of air pollution exposure." International journal of environmental research and public health 12.10 (2015): 12735-12760. 